Abstract

\title{
From Reagentless Biosensors to Biofuel Cells and Self-Powered Bioelectrochemical Devices ${ }^{\dagger}$
}

\author{
Wolfgang Schuhmann
}

Analytical Chemistry-Center for Electrochemical Sciences (CES), Ruhr-Universität Bochum, Universitätsstr. 150, 44780 Bochum, Germany; wolfgang.schuhmann@rub.de

+ Presented at the 5th International Symposium on Sensor Science (I3S 2017), Barcelona, Spain, 27-29 September 2017.

Published: 20 November 2017

Coupling of biocatalytic redox reactions with electrode surfaces is, on the one hand, the basis for the design of targeted biosensors, the electrochemical readout of DNA assays and, on the other hand, the basis for harvesting energy using biomolecules as recognition elements and catalysts. Due to the fact that the redox centers are often deeply buried within the protein structure of suitable enzymes, wiring of the enzyme integrated redox sites to the electrode surface is of utmost importance.

This presentation focuses on recent developments in:

1. Wiring of enzymes using designed redox polymers

2. Design of biofuel cells with increased open-circuit voltage

3. Self-powered biosensors and instrument-free substrate determination

4. Improving the power output of biofuel cells based on biosupercapacitors

Acknowledgments: With this contribution, I want to gratefully acknowledge the input of extraordinary coworkers and co-operation partners. Among those, I want specifically to thank Adrian Ruff, Sabine Alsaoub, Piyanut Pinyou, Felipe Conzuelo, Tim Bobrowski, Fangyuan Zhao, Francesca Lopez, Daliborka Jambrec, Magdalena Gebala, Ugur Kayran, Bianca Ciui, Sascha Pöller, Julian Szczesny, Nicolas Plumeré, and Sergey Shleev. This work was supported by the Cluster of Excellence RESOLV (EXC 1069) funded by the Deutsche Forschungsgemeinschaft (DFG) and the Deutsch-Israelische Projektkooperation (DIP) in the framework of the project "Nanoengineered Optobioelectronics with Biomaterials and Bioinspired Assemblies".

(C) 2017 by the authors. Licensee MDPI, Basel, Switzerland. This article is an open access article distributed under the terms and conditions of the Creative Commons Attribution (CC BY) license (http://creativecommons.org/licenses/by/4.0/). 